\title{
BEHCET'S DISEASE AND SEVERE REACTION TO 23-VALENT PNEUMOCOCCAL POLYSACCHARIDE VACCINE: A CASE REPORT
}

Lílian Santuza Santos Porto ${ }^{1,2}$, Cláudia Lopes Santoro Neiva ${ }^{1,2}$, Marcella Maria Soares Mello ${ }^{1}$, Luiza Ferreira Ribeiro Tadeuํㄹ , Izabela Guimarães Vieira Coelho ${ }^{1}$

1. Santa Casa de Misericórdia de Belo Horizonte, Belo Horizonte (MG), Brazil. 2. Rede Mater Dei de Saúde, Belo Horizonte (MG), Brazil.

*Corresponding author: lilianssporto@gmail.com

\section{BACKGROUND}

The European League Against Rheumatism (EULAR) recommends the 23-valent polysaccharide pneumococcal vaccine (23PPV) in patients with inflammatory rheumatic diseases. However, there are five published cases of systemic reactions to 23-PPV in patients with Behçet's disease (BD).

\section{CASE REPORT}

A 34-year-old female patient with history of oral (Fig. 1) and genital ulcers, erythema nodosum, pseudofolliculitis (Fig. 2) and arthritis came to the emergency department after an acute episode of aphasia and left hemiparesis. An urgent noncontrast computed tomography (CT) of the head showed a right median cerebral artery (MCA) distribution stroke. The patient was treated with alteplase and a mechanical thrombectomy was performed. During angiography, a critical stenosis of the right common carotid (CCA) and the right internal carotid (ICA) and the right MCA was showed. A stent was inserted in the CCA and ICA and a mechanical thrombectomy was carried out in the MCA. One month after discharge, she had convulsions and was taken to the neurology department. A brain magnetic resonance imaging (MRI) and a CT angiography was performed. No acute lesions were found, but there was a total occlusion of the right CCA and ICA stents (Fig. 3), although acetylsalicylic acid and clopidogrel had been used. She was referred to a rheumatologist, who diagnosed BD with vascular involvement, based on evidence of large vessel vasculitis and a history of oral and genital ulcers, erythema nodosum and arthritis. She was evaluated by a whole-body 18F-FDG-PET/CT, that was normal. Blood results showed normal CRP and ESR. The screening for thrombophilia and the immunological panel are normal. Colchicine, prednisone, azathioprine, pneumococcal and meningococcal vaccine were prescribed. One day after 23-PPV, the patient attended the emergency department with fever $\left(39^{\circ} \mathrm{C}\right)$, pain, redness, tenderness and swelling at the injection site. On arrival, her heart rate was $150 \mathrm{bpm}$ and blood pressure $80 / 50 \mathrm{mmHg}$. Blood tests showed leukocytosis and CPR $150 \mathrm{mg} / \mathrm{L}$. An ultrasound scan revealed only cellulitis. She was initially treated for sepsis. However, she responded very quickly, so her antibiotics were stopped. We believed that this was a reaction to her recent vaccine. This was further supported by two sets of negative blood cultures. Fifteen days after discharge, her arm reaction had settled and she started the immunosuppressive treatment.

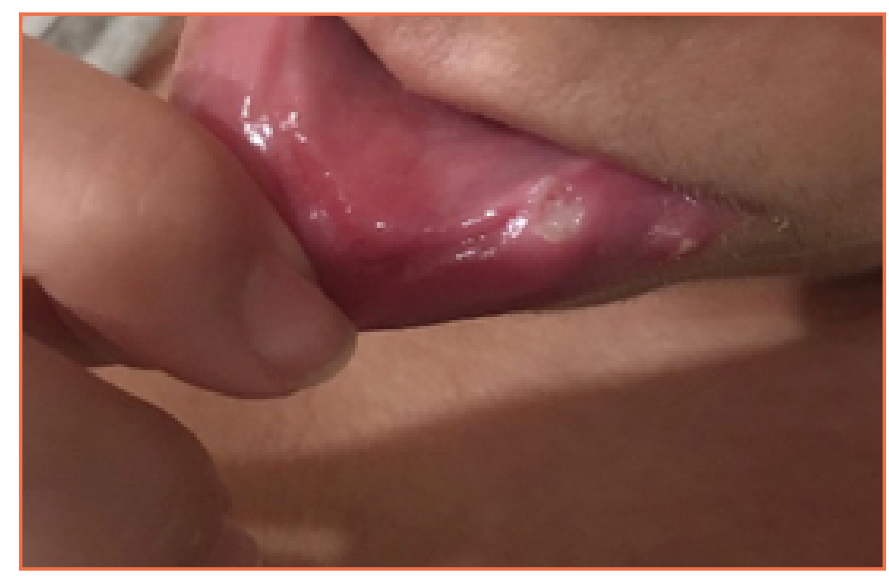

Figure 1. Oral ulcers. 


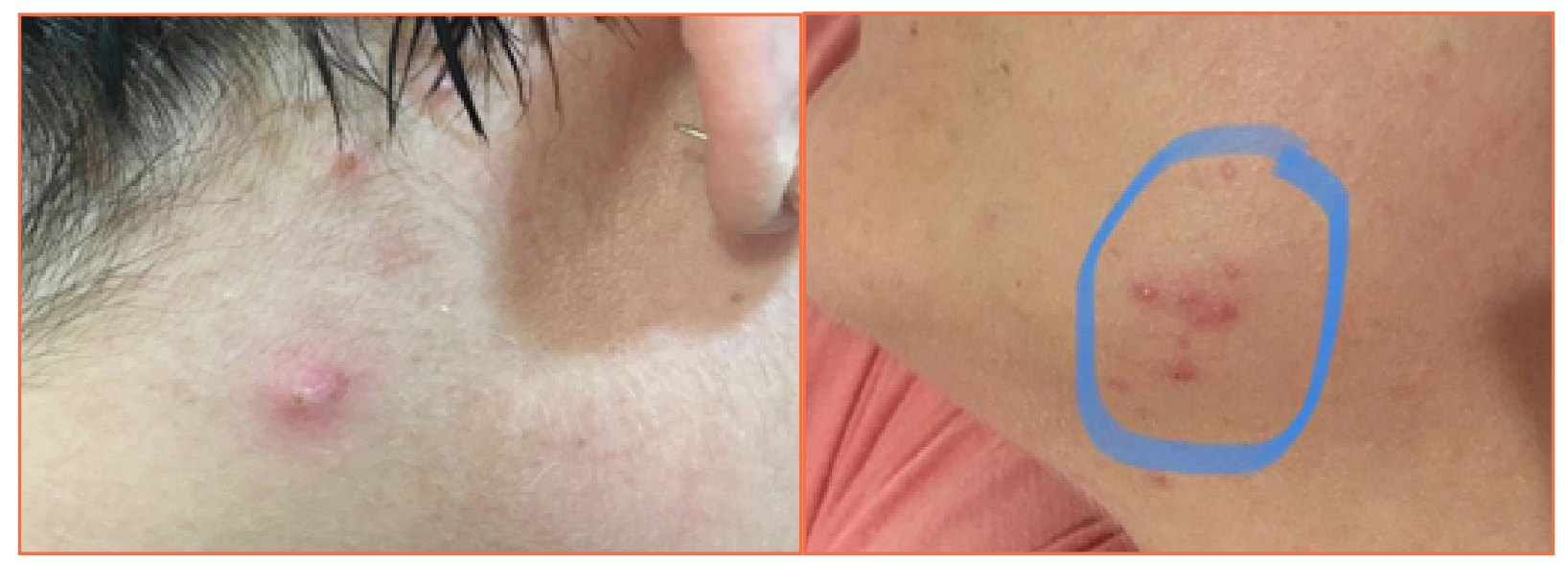

Figure 2. Pseudofolliculitis.

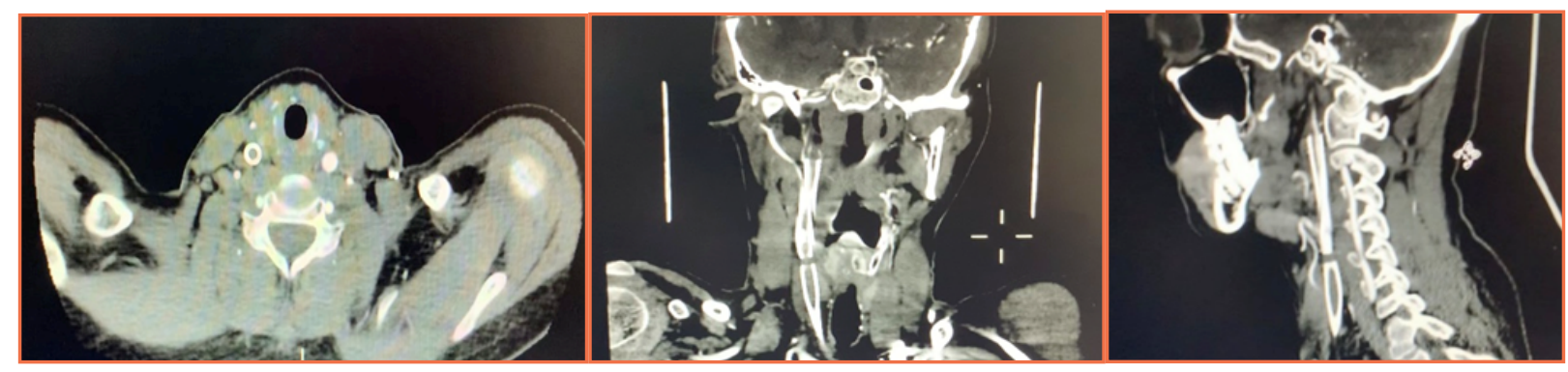

Figure 3. Transversal, coronal and sagittal view CT angiography of cervical vessels showing total occlusion of the right CCA and ICA stents.

\section{CONCLUSION}

Our case suggests that patients with BD are at risk of significant inflammatory reactions to 23-PPV. Further tests into the safety of 23-PPV in BD patients are needed. 